\title{
De novo production of six key grape aroma monoterpenes by a geraniol synthase-engineered $S$. cerevisiae wine strain
}

\author{
Ester Pardo' ${ }^{1}$ Juan Rico ${ }^{1}$, José Vicente Gil ${ }^{1,2}$ and Margarita Orejas ${ }^{1 *}$
}

\begin{abstract}
Background: Monoterpenes are important contributors to grape and wine aroma. Moreover, certain monoterpenes have been shown to display health benefits with antimicrobial, anti-inflammatory, anticancer or hypotensive properties amongst others. The aim of this study was to construct self-aromatizing wine yeasts to overproduce de novo these plant metabolites in wines.

Results: Expression of the Ocimum basilicum (sweet basil) geraniol synthase (GES) gene in a Saccharomyces cerevisiae wine strain substantially changed the terpene profile of wine produced from a non-aromatic grape variety. Under microvinification conditions, and without compromising other fermentative traits, the recombinant yeast excreted geraniol de novo at an amount ( $750 \mu \mathrm{g} / \mathrm{L})$ well exceeding (>10-fold) its threshold for olfactory perception and also exceeding the quantities present in wines obtained from highly aromatic Muscat grapes. Interestingly, geraniol was further metabolized by yeast enzymes to additional monoterpenes and esters: citronellol, linalool, nerol, citronellyl acetate and geranyl acetate, resulting in a total monoterpene concentration $(\sim 1,558 \mu \mathrm{g} / \mathrm{L}) 230$-fold greater than that of the control. We also found that monoterpene profiles of wines derived from mixed fermentations were found to be determined by the composition of the initial yeast inocula suggesting the feasibility of producing 'à la carte' wines having predetermined monoterpene contents.
\end{abstract}

Conclusions: Geraniol synthase-engineered yeasts demonstrate potential in the development of monoterpene enhanced wines.

Keywords: Geraniol, Geraniol synthase, Metabolic engineering, Monoterpenes, Saccharomyces cerevisiae, Monoterpene bioconversion, Wine aroma, Self-aromatizing wine yeasts

\section{Background}

Aroma is one of the most appreciated traits in assessing wine quality, and among the hundreds of volatile compounds characterized only a small number influence its sensory perception (see $[1,2]$ and references therein). These aroma active compounds (e.g. terpenes, esters, alcohols) have their origins in grapes, the metabolism of microorganisms (especially the wine-making yeast

\footnotetext{
*Correspondence: morejas@iata.csic.es

${ }^{1}$ Instituto de Agroquímica y Tecnología de Alimentos, Consejo Superior de Investigaciones Científicas (IATA-CSIC), Agustín Escardino 7, 46980 Paterna, Valencia, Spain

Full list of author information is available at the end of the article
}

Saccharomyces cerevisiae), and wine aging and storage conditions.

Monoterpenes ( $\mathrm{a} \mathrm{C}_{10}$ class of terpenes mainly derived from grapes) are key odorants associated with the varietal (or primary) aromas of certain white wines. Linalool, geraniol, nerol, citronellol and $\alpha$-terpineol are the major constituents of aromatic grape varieties (e.g. Muscat d'Alexandrie, Gewürztraminer, Riesling), imparting floral and fruity attributes (reviewed in $[3,4]$ ), and certain dietary monoterpenes are of nutraceutical importance because of their antimicrobial, antiviral, anti-proliferative, antioxidative, anxiolytic, hypotensive or anti-inflammatory properties, among other activities (see [5-8] and references therein). Apart from the natural properties of 
a grape variety, monoterpene content is also influenced by uncontrollable factors such as climate and soil. A large proportion of these monoterpenes is present in grape musts as non-volatile odorless sugar glycoconjugates that can be released enzymatically using industrial glycosidase cocktails or recombinant wine yeast strains expressing such activities (for reviews see [9-11]). Nevertheless, a number of grape varieties are aromatically 'neutral' and almost completely lack free monoterpenes and their precursors [4]. Thus there is considerable variability in monoterpene contents in grapes.

Monoterpene biosynthesis in plants is effected by monoterpene synthases (MTPSs). Many of their corresponding genes have been characterized [12, 13] and considerable expansion of these has been observed in grapevine (Vitis vinifera) [14, 15]. S. cerevisiae wine strains themselves produce only tiny quantities of monoterpenes (e.g. up to 1.2 or $4 \mu \mathrm{g} / \mathrm{L}$ of geraniol and linalool, respectively) [16] because they lack MTPSs and cannot therefore contribute to ameliorating monoterpene deficiency in grape must. Notwithstanding the non-acceptability of GMOs, especially by European wine consumers and industries, vinification by engineered monoterpene-producing wine yeast strains could thus constitute a means to enhance varietal wine aroma. In this regard, successful expression of the Clarkia breweri S-linalool synthase $(L I S)$ gene in a $S$. cerevisiae wine yeast strain has provided proof of concept by virtue of de novo production of linalool in wines to about $19 \mu \mathrm{g} / \mathrm{L}$ [17]. This metabolic manipulation was possible because plant MTPSs catalyze the synthesis of monoterpenes from geranyl pyrophosphate (GPP) in a single step, and S. cerevisiae has enough free GPP (an intermediate in ergosterol biosynthesis) under vinification conditions to be used as a substrate by these plant enzymes. In addition $S$. cerevisiae has the ability to metabolize supplemented monoterpenes, the bioconversions of (i) geraniol into citronellol, linalool, nerol and geranyl acetate, (ii) nerol into geraniol, linalool and $\alpha$-terpineol, (iii) linalool into $\alpha$-terpineol and (iv) citronellol into citronellyl acetate having been reported (see [18-20] and references therein). Thus an engineered monoterpene-producing yeast could also play a valuable additional role in the development of wine aroma by producing a wider spectrum of monoterpenes.

Previous work has shown that wine yeast strain $\mathrm{T}_{73}$ has a greater inherent capacity for recombinant monoterpene production compared to other laboratory and industrial wine strains [21]. Here we report the substantial modification of the terpene profile of a wine produced from a neutral grape variety using the $\mathrm{T}_{73}$ strain expressing the geraniol synthase (GES) gene from Ocimum basilicum (sweet basil) [22].

\section{Results and discussion}

Production of geraniol by a wine yeast strain expressing the GES gene of $O$. basilicum and its metabolic fate in synthetic defined (YPD) media

The truncated O. basilicum GES cDNA [22] (GenBank Accession No. AY362553) coding for a geraniol synthase lacking the first 34 codons-which encode the plastid transit peptide-was cloned under the control of the $S$. cerevisiae ACT1 (encoding actin) promoter $\left(A C T 1_{\mathrm{p}}\right)$ and the HIS3 (encoding imidazole glycerol-phosphate dehydratase) terminator (HIS3 $\left.3_{\mathrm{t}}\right)$ into the binary vector YEplac195 [23]. The resulting plasmid (YEp195Ges) was used to transform the $S$. cerevisiae $\mathrm{T}_{73}-4$ [24] wine strain and the uracil prototrophic $\left(\mathrm{ura}^{+}\right)$transformants YR377 and YR378 $\left(\mathrm{T}_{73} \mathrm{Ges}\right)$ were isolated. The growth rates of YR377, YR378 and the control strain YR70 $\left(\mathrm{T}_{73}-4\right.$ transformed with the empty plasmid) on liquid YPD media were almost identical, albeit slightly slower than that of the industrial strain $\mathrm{T}_{73}$ (Fig. 1a) as observed previously for other recombinant yeasts [17]. This indicates that neither the amount of geraniol nor the putative reduction of precursors from the isoprenoid pathway apparently produce deleterious effects on yeast growth under such conditions. In addition, GC and GC-MS analyses of these culture media showed similar extraordinarily high geraniol yields $(8,017.85 \pm 1,245.81$ and $7,859.12 \pm 1,614.62 \mu \mathrm{g} / \mathrm{L}$ after $32 \mathrm{~h}$ ) (Fig. 1b). These levels are about 16-fold higher than those produced by recombinant $S$. cerevisiae laboratory strains expressing the same GES gene, about 1.6-fold the amount produced by laboratory yeasts co-expressing GES and an optimized farnesyl diphosphate synthase [25, 26], and about 120-fold the amount of linalool excreted by engineered $\mathrm{T}_{73}-4$ wine strains expressing $L I S[17,21]$. These results clearly reinforce the previously shown importance of the genetic background of this industrial yeast for monoterpene production [21] but also that of the monoterpene synthase being expressed. In contrast to the $\mathrm{T}_{73} \mathrm{Lis}$ strains that produced linalool as the only end product, and in agreement with the reported ability of S. cerevisiae $\mathrm{T}_{73}$ to metabolise supplemented geraniol and its reaction products [20], the $\mathrm{T}_{73}$ Ges strains produced geraniol (84.83\%) and geraniol derivatives i.e. citronellol (10.92\%), nerol (3.90\%), linalyl acetate $(0.13 \%)$, geranyl acetate $(0.12 \%)$ and linalool $(0.1 \%)$. As expected, monoterpene production by the control strains lacking GES (YR70 and $\mathrm{T}_{73}$ ) was practically negligible $(7.13 \pm 1.12 \mu \mathrm{g} / \mathrm{L}$; $>1,300$-fold lower than YR377 and YR378) (Fig. 1b). YR377 was chosen for the microvinification experiments. 

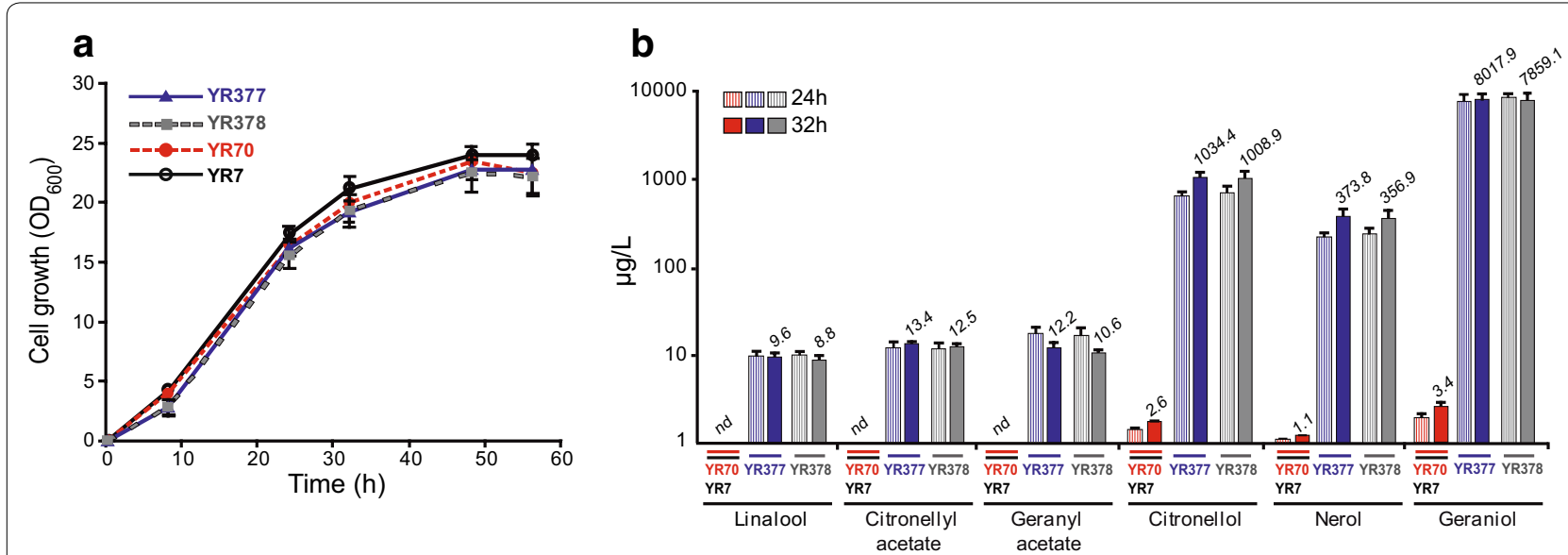

Fig. 1 Growth and monoterpene production in YPD of recombinant wine yeast $T_{73}-4$ expressing the 0 . basilicum GES gene. a Growth curves of $\mathrm{T}_{73}$ Ges (YR377 and YR378) and control strains YR70 ( $\mathrm{T}_{73}-4$ transformed with the empty plasmid) and $\mathrm{T}_{73}$. b Monoterpene production at 24 and $32 \mathrm{~h}$ by YR377, YR378 and controls. Numbers above the bars corresponding to $32 \mathrm{~h}$ indicate $\mu \mathrm{g} / \mathrm{L}$. Terpene concentrations are represented on a logarithmic scale. Results are presented as the mean and standard deviations of two independent assays with three replicates each.

\section{Aromatic wines from neutral grapes using the self-aromatizing wine yeast YR377}

Microvinification experiments were carried out in parallel on sterile Parellada white grape must using the wine yeast strain $\mathrm{T}_{73}-4$ that carries the GES expression cassette (YR377) and a control strain lacking GES (YR70). Both alcoholic fermentations progressed similarly (Fig. 2b) and reached completion in about 19 days leaving approximately 2 g/L residual sugar (i.e. dry wine). Given the persistence of the ura ${ }^{+}$phenotype (around 85\%) in YR377 and hence high maintenance of the GES expression cassette throughout the process it is evident that neither the expression of the GES gene nor its consequences affected the growth or fermentative capacity of the engineered wine strain.

To evaluate the influence of GES expression on wine aroma, volatile profiles were determined by GC and
GC-MS (Fig. 3a). As expected given the aromatic neutrality of the Parellada grape, free geraniol was undetectable in wines produced by YR70. In contrast, geraniol concentrations $(\sim 750 \mu \mathrm{g} / \mathrm{L})$ well in excess of its olfactory perception threshold (40-75 $\mu \mathrm{g} / \mathrm{L})$ and exceeding those present in wines obtained from the highly aromatic Muscat grapes (Additional file 1: Table S1) were found in wines fermented with the 'self-aromatizing' wine yeast YR377 (Fig. 3b; Table 1). Remarkably, GC analysis (Fig. 3) showed that apart from the geraniol peak there were also notable quantities $(\sim 810 \mu \mathrm{g} / \mathrm{L})$ of additional monoterpenes and esters associated with strain YR377: citronellol, linalool, nerol, citronellyl acetate and geranyl acetate, resulting in a total terpene concentration $>220$-fold greater than the control wine. With the exception of nerol and citronellyl acetate, the other compounds are present above their perception a

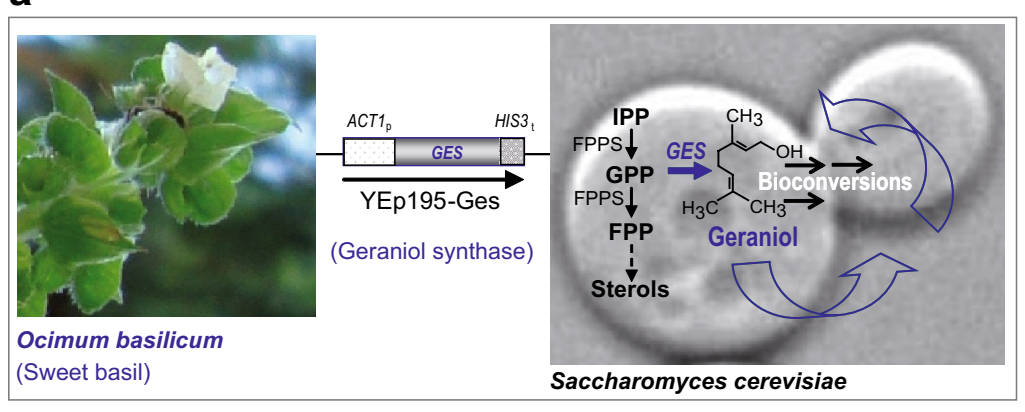

b

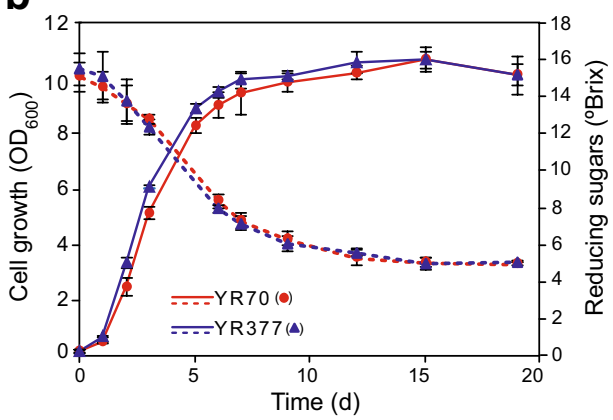

Fig. 2 Analyses of microvinifications. Microvinifications were carried out with YR377 ( $T_{73} G e s$ ) and the YR70 control strain transformed with the empty vector. a Schematic representation of the engineered mevalonate pathway in the $T_{73}$ Ges strains. IPP, isopentenyl pyrophosphate; GPP, geranyl pyrophosphate; FPP, farnesyl pyrophosphate; FPPS, FPP synthase. b Growth curves and kinetics of sugar consumption by YR377 and YR70 during the course of fermentations. Results are presented as the mean and standard deviation. 


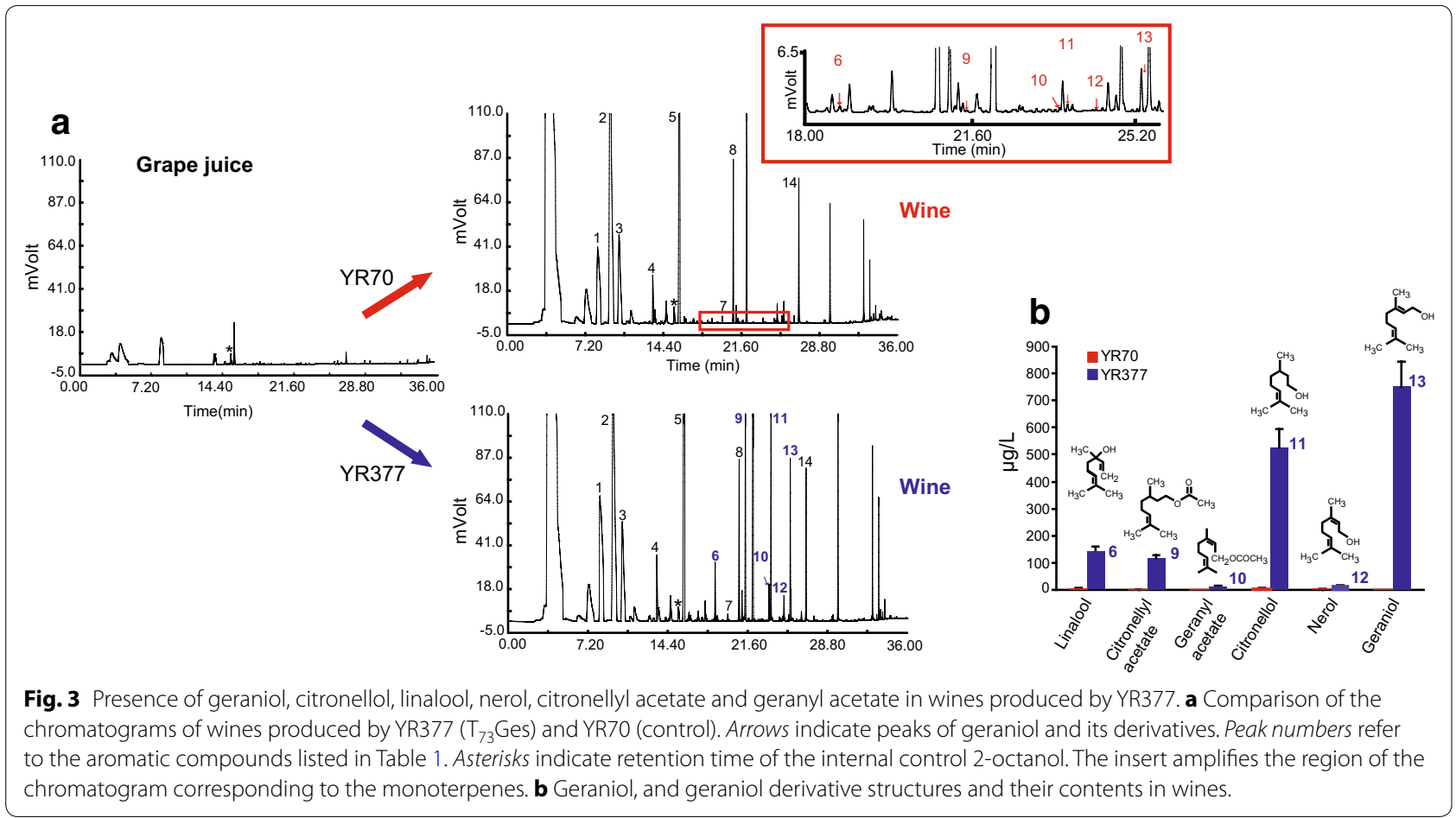

Table 1 Concentrations $(\mu \mathrm{g} / \mathrm{L})$, odor quality and thresholds of a selected subset of aromatic compounds found in Parellada wines fermented with the $\mathrm{T}_{73}$ Ges strain

\begin{tabular}{|c|c|c|c|}
\hline Compound $^{a}$ & Aroma descriptor ${ }^{b} / O T V s^{c}(\mu g / L)$ & YR70 $^{d}$ & YR377 $^{d}$ \\
\hline \multicolumn{4}{|l|}{ Monoterpenes } \\
\hline Linalool (6) & Floral/(6) & $2.36 \pm 0.41$ & $141.18 \pm 19.55$ \\
\hline Citronellyl acetate (9) & Fresh, fruity reminiscent of rose/(250) & $0.81 \pm 0.14$ & $116.32 \pm 12.23$ \\
\hline Geranyl acetate (10) & Pleasant, flowery reminiscent to rose lavender/(9) & nd & $12.84 \pm 1.51$ \\
\hline Citronellol (11) & Rose-like/(40) & $2.27 \pm 0.38$ & $526.35 \pm 67.17$ \\
\hline Nerol (12) & Fresh, sweet, rose-like/(300) & $1.33 \pm 0.06$ & $12.62 \pm 1.03$ \\
\hline Geraniol (13) & Rose-like/(40-75) & nd & $749.17 \pm 93.64$ \\
\hline$\alpha$-Terpineol & Lilac/(330-350) & nd & nd \\
\hline \multicolumn{4}{|l|}{ Alcohols } \\
\hline 3-Methyl 1 butanol (2) & Fusel oil, whiskey characteristic pungent/(250-300) & $79,376.67 \pm 12,716.18$ & $81,726.68 \pm 4,294.14$ \\
\hline 1-Hexanol (4) & Herbaceous, woody, sweet, green fruity/(2,500) & $343.79 \pm 93.98$ & $383.42 \pm 54.91$ \\
\hline 1-Octanol (7) & Fresh, orange-rose/(110-130) & $24.13 \pm 4.24$ & $27.21 \pm 3.59$ \\
\hline 2-Phenylethyl alcohol (14) & Rose-like $(750-1,100)$ & $24,524.45 \pm 3,065.82$ & $26,508.69 \pm 2,570.46$ \\
\hline \multicolumn{4}{|l|}{ Esters } \\
\hline Isoamyl acetate (1) & Fruity, banana, sweet, fragrant/(2) & $458.55 \pm 49.51$ & $484.51 \pm 79.92$ \\
\hline Ethyl caproate (3) & Fruity with a pineapple-banana note, winery/(1) & $190.52 \pm 21.36$ & $175.71 \pm 16.52$ \\
\hline Ethyl caprylate (5) & Pleasant, fruity, floral, wine-apricot note/(15) & $225.23 \pm 17.95$ & $178.66 \pm 23.60$ \\
\hline Ethyl decanoate (8) & Fruity reminiscent of grape (cognac)/(23-122) & $61.42 \pm 5.50$ & $56.46 \pm 6.60$ \\
\hline Total monoterpenes & & $6.77 \pm 0.99$ & $1,558.48 \pm 195.13$ \\
\hline
\end{tabular}

nd, not detected.

a Numbers between brackets refer to the GC peaks in Fig. 3.

b Aroma descriptors according to Feranoli's [35].

c Odor detection threshold values (OTVs) in water ( $\mu \mathrm{g} / \mathrm{L}$ ) were taken from the lists of Leffingwell \& Associates (http://www.leffingwell.com/).

${ }^{d}$ Values represent the means and standard deviation of two different microvinifications, three technical replications and two analytical replications. 
thresholds (Table 1). The presence of geraniol and its derivatives will enrich these wines with flowery and fruity notes.

GES and E. coli-expressed recombinant GES both exclusively catalyze the synthesis of geraniol from GPP [22]. Our comparative GC-MS data (Figs. 2, 3) revealed that the same terpenes found in wine were also excreted by YR377 when grown in synthetic (YPD) medium. Thus during vinification the enzymatic activities intrinsic to this wine yeast strain are also able to metabolize geraniol and its derivatives resulting in their conversion to other monoterpenes and aromatic esters, a situation resembling the metabolic diversion occurring in tomato expressing the GES gene [27]. The reduction of geraniol to citronellol and the acetylation of geraniol and citronellol are probably catalyzed by the oxidoreductase Oye 2 and the alcohol acetyl transferase Atf1 [28], respectively. An obvious strategy to further expand our ability to modulate wine aroma would therefore be to promote or suppress the formation of these geraniol derivatives by modification of these enzyme activities.

To investigate whether GES expression could lead to additional changes in a wine's volatile profile, determinations of other volatile compounds of oenological relevance were carried out on both recombinant yeast derived and control wines. The compositions and concentrations of higher alcohols (e.g. 2-phenylethyl alcohol) and acetate esters (e.g. isoamyl acetate), the presence of which is considered favorable for the aromatic properties of wines, were seen to be statistically similar in wines fermented with YR377 and YR70 strains (Table 1).

Introduction of the C. breweri LIS gene into wine yeast strain $\mathrm{T}_{73}-4$ ( $\left.\mathrm{T}_{73} \mathrm{Lis}\right)$ under the control of the TDH3 yeast promoter was our first attempt to construct a self-aromatizing wine yeast [17]. This resulted in de novo accumulation in wine of linalool alone to levels exceeding its odor perception threshold. Remarkably, the amount of geraniol-derived linalool produced by YR377 $\left(\mathrm{T}_{73} \mathrm{Ges}\right)$ was about 7.5 times greater than that obtained with $\mathrm{T}_{73} \mathrm{Lis}$ ( $\sim 141$ versus $\sim 19 \mu \mathrm{g} / \mathrm{L})$ and the total de novo terpene concentration is more than 80 -times greater, illustrating the importance of the MTPS employed in engineering strain $T_{73}$. These results justify the strategy of engineering the wine yeast isoprenoid pathway as a means of achieving efficient plant-derived aromatic monoterpene production during alcoholic fermentation.

\section{Mixed fermentation with $\mathrm{T}_{73}$ Ges and $S$. cerevisiae strains not producing monoterpenes serves to modulate levels of terpenes}

In order to assess whether it would be feasible to produce wines with a predetermined monoterpene content, vinifications were conducted using mixed starters $(1: 1)$ of yeast strains YR377 and YR70 and were compared to those obtained using pure cultures of YR377. The monoterpene profiles of wines derived from mixed fermentations were directly related to the composition of the initial inocula. Thus the amounts of geraniol $(\sim 388 \mu \mathrm{g} / \mathrm{L})$ and its derivatives $(\sim 311 \mu \mathrm{g} / \mathrm{L})$ detected were about half of those obtained using inocula of YR377 alone (Table 2).

Terpenes are also important flavor compounds in other fermented drinks. Geraniol, linalool and citronellol have all been shown to be important contributors to the floral, fruity and citrus flavors of beer [29], and biotransformations of these monoterpenes by ale and lager yeasts have been reported [19]. Engineered brewing-yeasts designed as vehicles for the de novo production of these monoterpenes thus have potential for use in the brewing industry. Moreover, certain monoterpenes have been shown to display a plethora of potential health benefits (see [5-8] and references therein).

\section{Conclusions}

These results demonstrate the considerable potential for geraniol-engineered yeasts in the development of wines with aromas à la carte'. Fermentation of grape musts with these and/or other yeast strains expressing novel plant MTPS genes and thus the possibility of producing monoterpenes absent from grapes will provide variety and novelty to the wine industry. Approaches including the manipulation of enzyme activities responsible for monoterpene bioconversions [28], the engineering of rate-limiting reactions in the mevalonate pathway [21] and/or the possibility of using diverse mixed starters to pre-determine monoterpene content could contribute to the enhancement of complexity in wine aroma (Fig. 4).

The work reported brings to the fore once again the question of whether modern genetic technologies, in this instance for improving wine yeasts, may become acceptable to industry and consumers given the continued

Table 2 Concentrations ( $\mu \mathrm{g} / \mathrm{L}$ ) of geraniol and derivatives found in Parellada wines co-fermented with GES strains

\begin{tabular}{lc}
\hline Monoterpenes & YR70:YR370 $^{\mathbf{a}}$ \\
\hline Linalool (6) & $57.19 \pm 7.76$ \\
Citronellyl acetate (9) & $40.68 \pm 3.32$ \\
Geranyl acetate (10) & $5.90 \pm 1.34$ \\
Citronellol (11) & $201.28 \pm 19.69$ \\
Nerol (12) & $5.53 \pm 0.18$ \\
Geraniol (13) & $388.35 \pm 8.96$ \\
Total & $698.93 \pm 41.25$
\end{tabular}

Numbers between brackets refer to the GC peaks in Fig. 3 .

a Values represent the means and standard deviation of two different microvinifications, three technical replications and two analytical replications. 


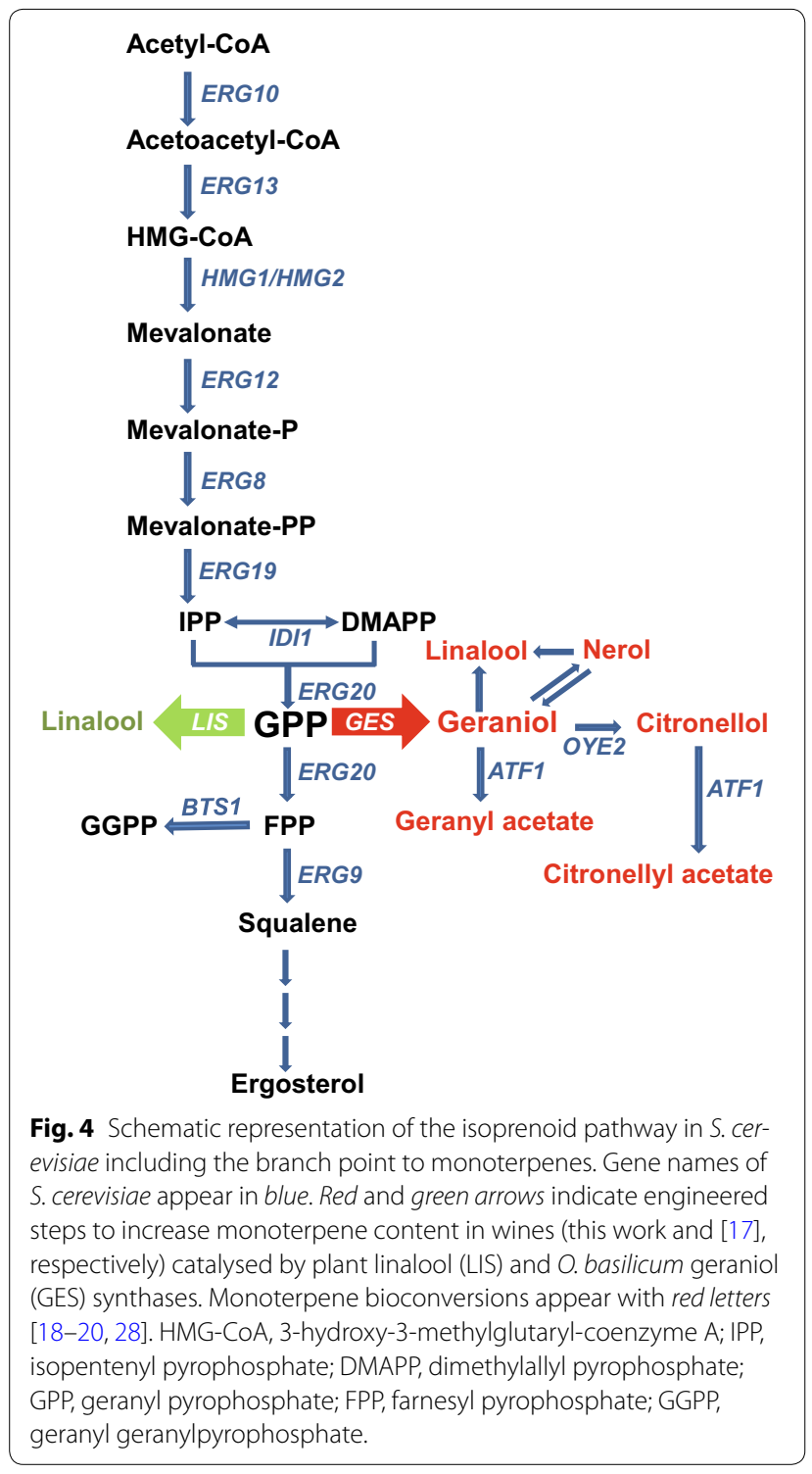

resistance to transgenic foods mainly in Europe. The advance reported in our study illustrates the biotechnological improvement of a food beyond the use of this type of technology for generating resistance to herbicides and pests via the genetic manipulation of a plant, and instead offers a clear alternative to transgenic grapes engineered to enhance free monoterpene content.

\section{Methods}

\section{Strains and culture conditions}

Escherichia coli DH5a [endA1, hsdR17, gyrA96, thi-1, relA1, supE44, recA1, $\Delta$ lacU169 (\$80 lacZ $\Delta M 15)]$ was used for cloning experiments and plasmid propagation. S. cerevisiae wine strain $\mathrm{T}_{73}-4$ [ura3::470/ura3::470] [24] (derived from $\mathrm{T}_{73}$, Lallemand) was used for GES expression. E. coli was maintained in LB medium (1\% tryptone,
$0.5 \%$ yeast extract, $1 \% \mathrm{NaCl}$ ) with or without $100 \mu \mathrm{g} / \mathrm{mL}$ ampicillin. S. cerevisiae strains were maintained in YPDrich medium (1\% yeast extract, $2 \%$ bacteriological peptone, $2 \%$ glucose $)$ or SD-minimal medium $(0.17 \%$ yeast nitrogen base without amino acids-Difco Laboratories, Detroit, USA-2\% glucose, $0.5 \%$ ammonium sulphate) with or without $20 \mathrm{mg} / \mathrm{L}$ uracil. For solid media, 1.5\% agar was added. To determine terpene yields of recombinant yeasts, aliquots from overnight cultures of selected transformants grown in SD medium lacking uracil were transferred to $250 \mathrm{~mL}$ flasks containing $50 \mathrm{~mL}$ of YPD medium at an initial $\mathrm{OD}_{600}$ of 0.05 . Yeast cultures were grown with continuous shaking $(200 \mathrm{rpm})$ at $30^{\circ} \mathrm{C}$ and aliquots of the cultures were taken at different times.

\section{Construction of yeast plasmids carrying the GES gene of $C$. breweri and yeast transformation}

The GES cDNA was obtained from pCRT7/CT-TOPO/ GES [22] via PCR as a 1.6-kb EcoRI [T4 DNA polymerase treated for blunt-ending]-BspLU11I fragment using the oligonucleotide pair GES-L35-Bs (5'-CCCACGCTACAT GTCTGCTTGCACGCCTTTGG-3'; BspLU11I is in italics and the artificial translation start site codon ATG and the GES-S35 TCT codon appear in bold) and GES-STOPRI (5'-CCCCCGAATTCTATTTATTGAGTGAAGAA GAGG-3'). The $H I S 3_{\mathrm{t}}$ was isolated as a $0.66-\mathrm{kb}$ HincII-SphI fragment obtained by PCR using genomic DNA of the $S$. cerevisiae strain FY1679 (MATa/MATa ura3-52/ura3-52) and the oligonucleotide pair His3_SalI (5'-AGGTC GACTAGTGACACCGATTATTTAAAGCTG-3 ${ }^{\prime}$ ) and His3_SphI (5'-AGGCATGCGAATTCGGATCCTCGGG GACACCAAATATGG-3'). These two fragments were subcloned downstream of $A C T 1_{\mathrm{p}}$ in the plasmid YEpACT4 [30] previously digested with NcoI and SphI, thereby generating plasmid YEp181Ges ( $2 \mu$; LEU2). The expression cassette $A C T 1_{\mathrm{p}}:: G E S:: H I S 3_{\mathrm{t}}$ was isolated from this plasmid as a 2.8-kb EcoRI fragment and subcloned into the same site of YEplac195 (2 $\mu$; URA3). The resulting plasmid (YEp195Ges) was used to transform the $S$. cerevisiae $\mathrm{T}_{73}-4$ [24] wine strain and uracil $\left(\mathrm{ura}^{+}\right)$prototrophic transformants $\left(\mathrm{T}_{73} \mathrm{Ges}\right)$ were thus isolated. To obtain the control strain YR70, $\mathrm{T}_{73}-4$ was transformed with YEplac195.

DNA manipulations were performed following standard protocols [31]. PCR fragments were individually cloned into the pGEM-T Easy vector (Promega) and the absence of mutations was confirmed by sequencing. Transformation of the $\mathrm{T}_{73}$ derived strain was done using lithium acetate to permeabilize the cells as previously described [24, 32]. Transformants were selected and maintained on SD plates without uracil. For plasmid stability analyses, transformants were grown under both selective (SD) and non-selective (YPD) conditions and the colonies growing under each condition were counted. 


\section{Microvinifications}

Two temporally independent microvinifications were done in triplicate at $20^{\circ} \mathrm{C}$ using $250 \mathrm{~mL}$ glass bottles containing $200 \mathrm{~mL}$ of Parellada white grape must (Villafranca del Penedés, Spain). The must ( ${ }^{\circ}$ Brix $\left.~ 15\right)$ was centrifuged and sterilized with $0.2 \%(\mathrm{v} / \mathrm{v})$ dimethyl dicarbonate (Velcorin; Bayer, Levercusen, Germany) and inoculated with $9 \times 10^{5}$ cells $/ \mathrm{ml}$ from overnight cultures of YR70 (uracil nutritional control) and YR377 $\left(\mathrm{T}_{73}\right.$ Ges). Samples were collected periodically to measure yeast growth and sugar consumption and thus monitor the progress of the fermentations. Sugar concentrations were initially measured as Brix grades using a Euromex RD. 5645 digital refractometer. After 15 days ${ }^{\circ}$ Brix stabilized to about 5 , and reducing sugar concentrations were measured using the Nelson-Somogyi method [33,34] to determine the end of the fermentations ('dry wine'; sugar concentration below $2 \mathrm{~g} / \mathrm{L}$ ). At this point (day 19), the persistence of the plasmids was measured (\% colonies grown on selective SD compared to those grown on complete YPD medium), the wines were centrifuged to remove yeast cells and then transferred to new bottles that were kept at $-20^{\circ} \mathrm{C}$ until their analysis.

\section{GC-MS analysis of volatiles}

Geraniol, geraniol derivatives and other volatiles were extracted and analyzed by headspace solid-phase microextraction (HS-SPME) using poly(dimethylsiloxane) (PDMS) fibers (Supelco, USA) coupled to gas chromatography (GC) and GC-mass spectrometry (MS) as previously reported [17]. 2-Octanol $(0.2 \mu \mathrm{g})$ was used as internal control. Identification of compounds was determined by comparing retention times and mass spectra with those of standards using a Thermo-Scientific model Focus-GC equipped with a HP-Innowax column (length $30 \mathrm{~m}$; inside diameter $0.25 \mathrm{~mm}$; film thickness $0.25 \mu \mathrm{m}$ ) and a Thermo Trace GC Ultra gas chromatograph coupled to a Thermo DSQ mass spectrometer (ThermoScientific), under the same chromatographic conditions. Ion spectra of the peaks of interest were identified by comparison with computerized libraries (e.g. Wiley6, NIST). The oven temperature was programmed as follows: $60^{\circ} \mathrm{C}$ for $5 \mathrm{~min}$, raised to $190^{\circ} \mathrm{C}$ at $5^{\circ} \mathrm{C} / \mathrm{min}$, then raised to $250^{\circ} \mathrm{C}$ at $20^{\circ} \mathrm{C} / \mathrm{min}$ and held $2 \mathrm{~min}$ at $250^{\circ} \mathrm{C}$. The injector temperature was $220^{\circ} \mathrm{C}$. Helium was the carrier gas at $1 \mathrm{~mL} / \mathrm{min}$ in the splitless mode. Compounds were quantified by integrating the peak areas of GC chromatograms.

\section{Additional file}

Additional file 1: Table S1. Concentrations ( $\mu \mathrm{g} / \mathrm{L}$ ) of fundamental monoterpenes found in wines made from different aromatic and neutral grape cultivars.

\section{Abbreviations}

GC: gas chromatography; GC-MS: gas chromatography-mass spectrometry; GES: geraniol synthase; GPP: geranyl pyrophosphate; LIS: Iinalool synthase; MTPS: monoterpene synthase; MVA: mevalonic acid; OTV: odour threshold value.

\section{Authors' contributions}

EP and JR carried out the experimental work and were involved in data analyses and interpretation of results. JVG participated in GC and GC-MS analyses. $\mathrm{MO}$ conceived, designed and coordinated the study, was involved in data analyses and interpretation of results and wrote the manuscript. All authors read and approved the final manuscript.

\section{Author details}

${ }^{1}$ Instituto de Agroquímica y Tecnología de Alimentos, Consejo Superior de Investigaciones Científicas (IATA-CSIC), Agustín Escardino 7, 46980 Paterna, Valencia, Spain. ${ }^{2}$ Departamento de Medicina Preventiva y Salud Pública, Ciencias de la Alimentación, Bromatología, Toxicología y Medicina Legal, Facultad de Farmacia, Universidad de Valencia, Vicente Andrés Estellés s/n, 46100 Burjassot, Valencia, Spain.

\section{Acknowledgements}

This work was funded by the Spanish MINECO/FEDER Grants CSD2007-0063 and AGL2011-29925. We also acknowledge support of the publication fee by the CSIC Open Access Publication Support Initiative through its Unit of Information Resources for Research (URICI). E. Pardo was financially supported by JCI2007-123/733 and JAEDOC086-FSE contracts. J. Rico was the recipient of a FPI (Formación de Personal de Investigador) pre-doctoral fellowship from the Comisión Interministerial de Ciencia y Tecnología (CICYT). We thank Dr. Eran Pichersky (University of Minesota) for the GES CDNA clone, Dr. Braulio Esteve (Universitat Rovira I Virgili) for Parellada must, Dr. Andrew P. MacCabe (IATA-CSIC) for critical reading of the manuscript, Dr. John Leffinwell for OTVs, and the Sequencing Core Facility of the University of Valencia for sequencing. The group also participated in the EC COST Action FA0907_BIOFLAVOUR.

\section{Compliance with ethical guidelines}

\section{Competing interests}

The authors declare that they have no competing interests.

Received: 24 April 2015 Accepted: 25 July 2015

Published online: 16 September 2015

\section{References}

1. Schreier P (1979) Flavor composition of wines: a review. CRC Crit Rev Food Sci Nutr 12:59-111

2. Polásková P, Herszage J, Ebeler SE (2008) Wine flavor: chemistry in a glass. Chem Soc Rev 37:2478-2489

3. Marais JS (1983) Terpenes in the aroma of grapes and wines: a review. Afr $J$ Enol Vitic 4:49-58

4. Mateo JJ, Jiménez M (2000) Monoterpenes in grape juice and wines. J Chromatogr 881:557-567

5. Duncan RE, El-Sohemy A, Archer MC (2005) Dietary factors and the regulation of 3-hydroxy-3-methylglutaryl coenzyme A reductase: implications for breast cancer development. Mol Nutr Food Res 49:93-100

6. Ajikumar PK, Tyo K, Carsen S, Mucha O, Phon TH, Stephanopoulos G (2008) Terpenoids: opportunities for biosynthesis of natural product drugs using engineered microorganisms. Mol Pharmaceut 19:167-190

7. Bohlmann J, Keeling Cl (2008) Terpenoid biomaterials. Plant J 54:656-669

8. Bastos JF, Moreira IJ, Ribeiro TP, Medeiros IA, Antoniolli AR, De Sousa DP et al (2009) Hypotensive and vasorelaxant effects of citronellol, a monoterpene alcohol, in rats. Basic Clin Pharmacol Toxicol 106:331-337

9. Maicas S, Mateo JJ (2005) Hydrolysis of terpenyl glycosides in grape juice and other fruit juices: a review. Appl Microbiol Biotechnol 67:322-335

10. Ramón D, Genovés S, Gil JV, Herrero O, MacCabe AP, Manzanares P et al (2005) Milestones in wine biotechnology. Minerva Biotech 17:33-45 
11. Cordente AG, Curtin CD, Varela CV, Pretorius IS (2012) Flavour-active wine yeast. Appl Microbiol Biotechnol 96:601-618

12. Degenhardt J, Köllner TG, Gershenzon J (2009) Monoterpene and sesquiterpene synthases and the origin of terpene skeletal diversity in plants. Phytochemistry 70:1621-1637

13. Chen F, Tholl D, Bohlmann J, Pichersky E (2011) The family of terpene synthases in plants: a mid-size family of genes for specialized metabolism that is highly diversified throughout the kingdom. Plant J 66:212-229

14. Jaillon O, Aury JM, Noel B, Policriti A, Clepet C, Casagrande A et al (2007) The grapevine genome sequence suggest ancestral hexaploidization in major angiosperm phyla. Nature 449:463-467

15. Martin DM, Aubourg S, Schouwey MB, Daviet L, Schalk M, Toub O et al (2010) Functional annotation, genome organization and phylogeny of the grapevine (Vitis vinifera) terpene synthase gene family based on genome assembly, FLcDNA cloning, and enzyme assays. J BMC Plant Biol $10: 226$

16. Carrau FM, Medina K, Boido E, Farina L, Gaggero C, Dellacassa E et al (2005) De novo synthesis of monoterpenes by Saccharomyces cerevisiae wine yeast. FEMS Microbiol Lett 243:107-115

17. Herrero O, Ramón D, Orejas M (2008) Engineering the Saccharomyces cerevisiae isoprenoid pathway for de novo production of aromatic monoterpenes in wine. Metab En 10:78-86

18. King A, Dickinson JR (2000) Biotransformation of monoterpene alcohols by Saccharomyces cerevisiae. Torulaspora delbrueckii and Kluyveromyces lactis. Yeast 16:499-506

19. King A, Dickinson JR (2003) Biotransformation of hop aroma terpenoids by ale and larger yeasts. FEMS Yeast Res 3:53-62

20. Gamero A, Manzanares P, Querol A, Belloch C (2011) Monoterpene alcohols release and bioconversion by Saccharomyces species and hybrids. Int J Food Microbiol 145:92-97

21. Rico J, Pardo E, Orejas M (2010) Enhanced production of a plant monoterpene by overexpression of the 3-hydroxy-3-methylglutaryl Coenzyme A reductase catalytic domain in Saccharomyces cerevisiae. Appl Environ Microbiol 76:6449-6454

22. lijima Y, Gang DR, Fridman E, Lewinsohn E, Pichersky E (2004) Characterization of geraniol synthase from peltate glands of sweet basil. Plant Physiol 134:370-379
23. Gietz RD, Sugino A (1988) New yeast-Escherichia coli shuttle vectors constructed with in vitro mutagenized yeast genes lacking six-base pair restriction sites. Gene 74:527-534

24. Puig S, Ramón D, Pérez-Ortín JE (1998) An optimized method to obtain stable food-safe recombinant wine strains. J Agric Food Chem 46:1689-1693

25. Oswald M, Fischer M, Dirninger N, Karst F (2007) Monoterpenoid biosynthesis in Saccharomyces cerevisiae. FEMS Yeast Res 7:413-421

26. Fischer MJC, Meyer S, Claudel P, Bergdoll M, Karst F (2011) Metabolic engineering of monoterpene synthesis in yeast. Biotechnol Bioeng 108:1883-1892

27. Davidovich-Rikanati R, Sitrit Y, Tadmor Y, lijima Y, Bilenko N, Bar E et al (2007) Enrichment of tomato flavour by diversion of the early plastidial terpenoid pathway. Nat Biotechnol 25:899-901

28. Steyer D, Erny C, Claudel P, Riveill G, Karst F, Legras JL (2012) Genetic analysis of geraniol metabolism during fermentation. Food Microbiol 33:228-234

29. Takoy K, Itoga Y, Koie K, Kosugi T, Shimase M, Katayama Y et al (2010) The contribution of geraniol metabolism to the citrus flavour of beer: synergy of geraniol and $\beta$-citronellol under coexistence with excess linalool. J Inst Brew 116:251-260

30. Sánchez-Torres P, González-Candelas L, Ramón D (1998) Heterologous expression of a Candida molischiana anthocyanin-beta-glucosidase in a wine yeast strain. J Agric Food Chem 46:354-360

31. Sambrook J, Russell DE (2001) Molecular cloning: a laboratory manual, 3rd edn. Cold Spring Harbor Laboratory Press, Cold Spring Harbor

32. Gietz RD, Schiestl RH, Willems AR, Woods RA (1995) Studies on the transformation of intact yeast cells by the LiAC/SS-DNA/PEG procedure. Yeast 11:355-360

33. Nelson N (1944) A photometric adaptation of the Somogyi method for the determination of glucose. J Biol Chem 153:375-380

34. Somogyi M (1952) Notes on sugar determination. J Biol Chem 195:19-23

35. Burdock GA (2002) Feranoli's handbook of flavor ingredients, 4th edn. CRC Press, Florida

\section{Submit your next manuscript to BioMed Central and take full advantage of:}

- Convenient online submission

- Thorough peer review

- No space constraints or color figure charges

- Immediate publication on acceptance

- Inclusion in PubMed, CAS, Scopus and Google Scholar

- Research which is freely available for redistribution

Submit your manuscript at 